\title{
NEAR-RESONANT REGIMES OF A MOVING LOAD ON A PRE-STRESSED INCOMPRESSIBLE ELASTIC HALF-SPACE
}

\author{
Askar KUDAIBERGENOV*, Askat KUDAIBERGENOV*, Danila PRIKAZCHIKOV"****
}

\author{
*Department of Mathematical and Computer Modelling, Faculty of Mechanics and Mathematics, \\ Al-Farabi Kazakh National University, 71 Al-Farabi Ave., 050040, Almaty, Kazakhstan \\ * School of Computing and Mathematics, Keele University, Keele, Staffordshire, ST5 5BG, UK \\ ${ }^{* * *}$ Institute for Problems in Mechanical Engineering RAS, 61 Bolshoy Pr., Saint-Petersburg, 199178, Russia
}

askar.kudaibergenov@kaznu.kz, askat.kudaibergenov@kaznu.kz, d.prikazchikov@keele.ac.uk

received 15 October 2020, revised 14 April 2021, accepted 19 April 2021

\begin{abstract}
The article is concerned with the analysis of the problem for a concentrated line load moving at a constant speed along the surface of a pre-stressed, incompressible, isotropic elastic half-space, within the framework of the plane-strain assumption. The focus is on the near-critical regimes, when the speed of the load is close to that of the surface wave. Both steady-state and transient regimes are considered. Implementation of the hyperbolic-elliptic asymptotic formulation for the surface wave field allows explicit approximate solution for displacement components expressed in terms of the elementary functions, highlighting the resonant nature of the surface wave. Numerical illustrations of the solutions are presented for several material models.
\end{abstract}

Key words: moving load, incompressible, pre-stress, asymptotic

\section{INTRODUCTION}

Moving loads on an elastic half-space have been the subject of numerous investigations, motivated by important engineering applications related to ground vibrations caused by moving transport vehicles; for example, refer to studies by Krylov (1996) and Cao et al. (2012). In the classical contribution of Cole and Huth (1958), a steady-state solution for an elastic half-plane subject to a moving load was obtained. It is worth mentioning that the resonant nature of the Rayleigh wave may have been noticed in this early article, see also the study by Goldstein (1965). A substantial part of consideration for moving loads is focused on steady-state regimes, refer e.g., recent results for porous anisotropic half-space by Wang et al. (2021) and the study for a thermoelastic half-space with double porosity by Kumar and Vohra (2020). We also note the articles dealing with time-harmonic moving loads; refer to studies by Lefeuve-Mesgouez et al. (2000) and Sun et al. (2019), and interesting aspects of transition when surface load moves over the interface of two elastic materials; refer to the study by van Dalen et al. (2015). There are relatively few treatments of transient modes in moving load problems, including early works (Payton, 1967; Gakenheimer and Miklowitz, 1969) and also more recent contributions (de Hoop, 2002; Kaplunov et al., 2010b). It is known that an analysis of transient dynamics is generally non-trivial, often requiring a numerical approach; refer to studies by Bratov (2011) and Smirnov et al. (2012). We also mention active studies of moving loads on elastic structures, for example refer to a textbook (Fryba, 1999) and references therein, as well as recent works in studies by Dimitrovová (2017), Wang et al. (2020) and Lu et al. (2020).

This article relies on a recent approach to near-resonant regimes of the moving load on an elastic half-space, originating from the hyperbolic-elliptic models for surface waves; refer to the study by Kaplunov and Prikazchikov (2017). Models consist of elliptic equations associated with decay into the interior, along with hyperbolic equations on the surface governing wave propagation. This methodology has allowed a number of explicit approximate solutions of moving load problems; refer to studies by Kaplunov et al. (2010a), Kaplunov et al. (2013), Erbaş et al. (2017), and Ege et al. (2017). The advantage of this approach is related to the representation of the surface wave field in terms of a single harmonic function, providing reduction of the vector problem of elastodynamics to a scalar formulation. Recent developments in this area include the incorporation of effects of anisotropy (Fu et al., 2020), a refined second-order model (Wootton et al., 2020), explicit formulations for seismic meta-surfaces in the form of an array of resonators attached to the surface (Ege et al., 2018; Wootton et al., 2019) and formulations for surface wave on a coated halfspace with non-classical boundary conditions (Kaplunov et al., 2019).

The hyperbolic-elliptic plane-strain model for surface wave on a pre-stressed incompressible elastic half-space has been derived in the study by Khajiyeva et al. (2018), allowing a scalar formulation for the surface wave field induced by prescribed surface stresses. In this work, we implement these results to analyse the near-critical regimes for the line force moving at a constant speed along the surface. Both steady-state and transient problems are considered. As a result, explicit expressions for the displacement field are obtained in terms of elementary functions, confirming the resonant nature of surface wave speed. In case of transient displacements, distinction between the sub-critical, super-critical and resonant regimes follows immediately from the analysis on the surface. Then, using Poisson's formula, solution is restored over the interior. Consideration of large time limit allows approxima- 
tions for the components of rigid body motion. The obtained results are illustrated numerically for several material models, including the neo-Hookean, Gent, and Gent-Gent strain-energy functions.

\section{STATEMENT OF THE PROBLEM}

Consider a homogeneous incompressible elastic body with an initial state $B_{0}$ in the domain $X_{2} \geq 0$. Under the action of a homogeneous static deformation $x_{i}=x_{i}\left(X_{A}\right)$, the body transforms to a finitely deformed equilibrium state $B_{e}$, which corresponds to a half-space $X_{2} \geq 0$, and after superimposing infinitesimal timedependent motion $u_{i}\left(x_{j}, t\right)$, it moves to the current state $B_{t}$ with the position vector $\bar{x}_{l}\left(X_{A}, t\right)$ given by:

$\bar{x}_{l}\left(X_{A}, t\right)=x_{i}\left(X_{A}\right)+u_{i}\left(x_{j}, t\right)$.

The equations provided below address the plane-strain problem, for which $u_{3}=0$ and $u_{1}=u_{1}\left(x_{1}, x_{2}, t\right), \quad u_{2}=$ $u_{2}\left(x_{1}, x_{2}, t\right)$, giving the following coupled equations of incremental motion:

$A_{1111} \frac{\partial^{2} u_{1}}{\partial x_{1}^{2}}+\left(A_{1122}+A_{1221}\right) \frac{\partial^{2} u_{2}}{\partial x_{1} \partial x_{2}}+A_{2121} \frac{\partial^{2} u_{1}}{\partial x_{2}^{2}}-\frac{\partial p_{t}}{\partial x_{1}}=$ $\rho \frac{\partial^{2} u_{1}}{\partial t^{2}}$

$A_{1212} \frac{\partial^{2} u_{2}}{\partial x_{1}^{2}}+\left(A_{1122}+A_{1221}\right) \frac{\partial^{2} u_{1}}{\partial x_{1} \partial x_{2}}+A_{2222} \frac{\partial^{2} u_{2}}{\partial x_{2}^{2}}-\frac{\partial p_{t}}{\partial x_{2}}=$ $\rho \frac{\partial^{2} u_{2}}{\partial t^{2}}$

where $A_{i j k l}$ are the components of the fourth-order elasticity tensor (Ogden, 1984), $\rho$ is mass density and $p_{t}$ is the timedependent incremental component of pressure associated with the incompressibility constraint whose linearised measure is given by:

$\frac{\partial u_{1}}{\partial x_{1}}+\frac{\partial u_{2}}{\partial x_{2}}=0$.

In this article, we focus on studying the effect of a vertical load, represented as a concentrated line force moving at a constant speed $v$, on the pre-stressed elastic half-space (refer Fig. 1). Then, the boundary conditions on the surface $x_{2}=0$ are given in the following form:

$A_{2121} \frac{\partial u_{1}}{\partial x_{2}}+\left(A_{1221}+\bar{p}\right) \frac{\partial u_{2}}{\partial x_{1}}=0$,

$A_{1122} \frac{\partial u_{1}}{\partial x_{1}}+\left(A_{2222}+\bar{p}\right) \frac{\partial u_{2}}{\partial x_{2}}-p_{t}=P_{0} \delta\left(x_{1}-v t\right)$,

where $\bar{p}=A_{2121}-A_{1221}-\sigma_{2}$ is static pressure in the equilibrium state $B_{e}, \sigma_{2}$ is the normal Cauchy stress component, $\delta$ is the Dirac delta function and $P_{0}$ is the amplitude.

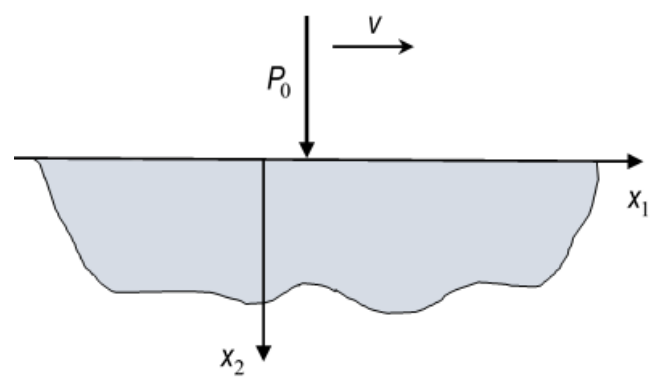

Fig. 1. Pre-stressed elastic half-space under the effect of a moving load
We consider the near-resonant regime, when the speed of the moving load is close to surface wave speed, thus the contribution of surface wave dominates over that of the bulk waves.

Introducing the auxiliary harmonic function $\psi_{1}$ [see Khajiyeva et al. (2018) for details], the displacements may be expressed as follows:

$u_{1}\left(x_{1}, x_{2}, t\right)=\frac{\partial \psi_{1}\left(x_{1}, k_{1} x_{2}, t\right)}{\partial x_{2}}+\vartheta \frac{\partial \psi_{1}\left(x_{1}, k_{2} x_{2}, t\right)}{\partial x_{2}}$,

$u_{2}\left(x_{1}, x_{2}, t\right)=-\frac{\partial \psi_{1}\left(x_{1}, k_{1} x_{2}, t\right)}{\partial x_{1}}-\vartheta \frac{\partial \psi_{1}\left(x_{1}, k_{2} x_{2}, t\right)}{\partial x_{1}}$,

where $\vartheta=\frac{\gamma\left(k_{1}^{2}+1\right)-\sigma_{2}}{\gamma\left(k_{2}^{2}+1\right)-\sigma_{2}}$, and $k_{1}, k_{2}$ are related by:

$k_{1}^{2}+k_{2}^{2}=\frac{2 \beta-\rho c_{R}^{2}}{\gamma}, \quad k_{1}^{2} k_{2}^{2}=\frac{\alpha-\rho c_{R}^{2}}{\gamma}$,

With:

$\alpha=A_{1212}, \quad 2 \beta=A_{1111}+A_{2222}-2\left(A_{1122}+A_{1221}\right)$,

$\gamma=A_{2121}$,

and $c_{R}$ denoting surface wave speed, being the solution of

$\gamma\left(\alpha-\rho c_{R}^{2}\right)+\left(2 \beta+2 \gamma-2 \sigma_{2}-\rho c_{R}^{2}\right) \sqrt{\gamma\left(\alpha-\rho c_{R}^{2}\right)}=$ $\left(\gamma-\sigma_{2}\right)^{2}$,

refer to the study by Dowaikh and Ogden (1990).

The approximate formulation of the original problem in elasticity as defined in Eqs (2)-(4), oriented towards the surface wave field, has been developed in the study by Khajiyeva et al. (2018), reducing the vector problem in elasticity to a scalar problem for the elliptic equation in respect of the potential $\psi_{1}$. The methodology of the derivation relies on the slow-time perturbation procedure, extending the previous results for isotropic elasticity; see Kaplunov and Prikazchikov (2017). The resulting hyperbolicelliptic model for surface wave field in a pre-stressed incompressible elastic half-space excited by the vertical surface loading $f_{2}=f_{2}\left(x_{1}, t\right)$ is formulated in terms of the potential $\psi_{1}$ as an elliptic equation:

$\frac{\partial^{2} \psi_{1}}{\partial x_{2}^{2}}+k_{1}^{2} \frac{\partial^{2} \psi_{1}}{\partial x_{1}^{2}}=0$,

which is subject to the boundary condition on the surface, given by a hyperbolic equation [compare with formula (39) in the study by Khajiyeva et al. (2018)]

$\frac{\partial^{2} \psi_{1}}{\partial x_{1}^{2}}-\frac{1}{c_{R}^{2}} \frac{\partial^{2} \psi_{1}}{\partial t^{2}}=-\frac{2 a_{11} f_{2}^{*}}{c_{R}\left(a_{21} b_{1}-a_{11} b_{2}\right)}$ at $x_{2}=0$,

where:

$$
\begin{aligned}
& \begin{aligned}
\begin{array}{l}
a_{11}=\gamma\left(k_{1}^{2}+1\right) \\
-
\end{array} \sigma_{2}, \quad a_{21} \\
=k_{1}\left(2 \beta-\rho c_{R}^{2}-\sigma_{2}-\gamma\left(k_{1}^{2}-1\right)\right)
\end{aligned} \\
& f_{2}=P_{0} \delta\left(x_{1}-v t\right), \quad b_{1}=\frac{2 \rho c_{R}}{k_{2}^{2}-k_{1}^{2}}\left(k_{1}^{2}-1-\vartheta\left(k_{2}^{2}-1\right)\right), \\
& b_{2}=g_{1}+\vartheta g_{2}, \\
& g_{j}=\frac{\rho c_{R}\left(k_{j}^{2}-1\right)}{\gamma k_{j}\left(k_{m}^{2}-k_{j}^{2}\right)}\left(2 \beta-\rho c_{R}^{2}-\sigma_{2}+\gamma\left(1-3 k_{j}^{2}\right)\right) \\
& \quad-2 k_{j} \rho c_{R}
\end{aligned}
$$

$j, m=1,2 ; j \neq m$ and the asterisk denotes the Hilbert integral transform. In the forthcoming section, we adapt this asymptotic formulation (7) and (8) to the considered moving load problem. 


\section{EXPLICIT STEADY-STATE SOLUTION FOR THE NEAR-RESONANT REGIME}

For the sake of convenience, the asymptotic models (7) and (8) can be expressed in terms of the harmonic conjugate of $\psi_{1}$. The elliptic Eq. (7) becomes:

$\frac{\partial^{2} \psi_{1}^{*}}{\partial x_{2}^{2}}+k_{1}^{2} \frac{\partial^{2} \psi_{1}^{*}}{\partial x_{1}^{2}}=0$,

and the hyperbolic Eq. (8) is rewritten as:

$\frac{\partial^{2} \psi_{1}^{*}}{\partial x_{1}^{2}}-\frac{1}{c_{R}^{2}} \frac{\partial^{2} \psi_{1}^{*}}{\partial t^{2}}=P_{1} \delta\left(x_{1}-v t\right)$ at $x_{2}=0$,

where $P_{1}=\frac{2 a_{11} P_{0}}{c_{R}\left(a_{21} b_{1}-a_{11} b_{2}\right)}$.

Now we consider the steady-state regime and introduce a moving coordinate, which can be represented as follows:

$\xi=x_{1}-v t$.

Then, Eq. (10) takes the form-

$\left(1-\frac{v^{2}}{c_{R}^{2}}\right) \frac{\partial^{2} \psi_{1}^{*}}{\partial \xi^{2}}=P_{1} \delta(\xi)$.

On integrating the latter, we have-

$\frac{\partial \psi_{1}^{*}}{\partial \xi}=-\frac{c_{R}^{2} P_{1}}{v_{-} v_{+}}\left(H(\xi)-\frac{1}{2}\right)$,

where the constant of integration is chosen according to the symmetry rule as it cannot be determined by considering the steadystate regime (Kaplunov and Prikazchikov, 2017), and $v_{ \pm}=v \pm$ $c_{R}$.

Hence, restoring the solution into the interior, we obtain-

$\frac{\partial \psi_{1}^{*}\left(\xi, k_{1} x_{2}\right)}{\partial \xi}=-\frac{c_{R}^{2} P_{1}}{\pi v_{-} v_{+}} \tan ^{-1} \frac{\xi}{k_{1} x_{2}}$

from which the harmonic conjugate function can be found as-

$\frac{\partial \psi_{1}\left(\xi, k_{1} x_{2}\right)}{\partial \xi}=\frac{c_{R}^{2} P_{1}}{2 \pi v_{-} v_{+}} \ln \left(\xi^{2}+k_{1}^{2} x_{2}^{2}\right)$.

Thus, on substituting (14) and (15) into (5), the displacements $u_{1}$ and $u_{2}$ are given explicitly by:

$u_{1}=\frac{c_{R}^{2} P_{1}}{\pi v_{-} v_{+}}\left[k_{1} \tan ^{-1} \frac{\xi}{k_{1} x_{2}}+\vartheta k_{2} \tan ^{-1} \frac{\xi}{k_{2} x_{2}}\right]$,

$u_{2}=-\frac{c_{R}^{2} P_{1}}{2 \pi v_{-} v_{+}}\left[\ln \left(\xi^{2}+k_{1}^{2} x_{2}^{2}\right)+\vartheta \ln \left(\xi^{2}+k_{2}^{2} x_{2}^{2}\right)\right]$

The resonant nature of surface wave speed is clearly observed from the solution available in Eq. (16).

\section{TRANSIENT MOVING LOAD PROBLEM}

Now let us consider a transient problem. Within this article, we rely on the approach presented in the study by Kaplunov et al. (2010a), with the solution of the hyperbolic Eq. (10) written as a convolution of the right-hand side with the fundamental solution, namely:

$\psi_{1}^{*}(\xi, 0, t)=\frac{c_{R} P_{1}}{2} \int_{0}^{t}\left(H\left(\xi+v_{-} r\right)-H\left(\xi+v_{+} r\right)\right) d r$,

where $H$ is the Heaviside function.

The form of the integral in (17) motivates separate study of three regimes, including the sub-Rayleigh $\left(v<c_{R}\right)$, superRayleigh $\left(v>c_{R}\right)$ and the resonant one $\left(v=c_{R}\right)$.

Introducing $\varphi=-\frac{2}{c_{R} P_{1}} \psi_{1}^{*}$ for convenience, we obtain for

a) sub-Rayleigh regime $\left(v<c_{R}\right)$ :

$\varphi(\xi, 0, t)=\left\{\begin{array}{cc}\frac{\xi}{v_{-}}+t, & 0 \leq \xi<-v_{-} t, \\ \frac{\xi}{v_{+}}+t, & -v_{+} t<\xi<0, \\ 0, & \text { otherwise }\end{array}\right.$

b) super-Rayleigh regime $\left(v>c_{R}\right)$ :

$\varphi(\xi, 0, t)=\left\{\begin{array}{c}\xi\left(\frac{1}{v_{+}}-\frac{1}{v_{-}}\right),-v_{-} t \leq \xi \leq 0, \\ \frac{\xi}{v_{+}}+t,-v_{+} t<\xi<-v_{-} t, \\ 0, \text { otherwise }\end{array}\right.$

c) resonant regime $\left(v=c_{R}\right)$ :

$\varphi(\xi, 0, t)=\left\{\begin{array}{c}\frac{\xi}{2 c_{R}}+t, \quad-2 c_{R} t \leq \xi \leq 0, \\ 0, \text { otherwise }\end{array}\right.$

Now, once the solution has been found on the surface $x_{2}=0$ in terms of the function $\varphi$, we can conduct the analysis with depth, i.e. restore the solution over the interior of the half-space $x_{2}>0$. Using the elliptic Eq. (9) and applying Poisson's formula, the potential $\psi_{1}^{*}$ is expressed as

$\psi_{1}^{*}\left(\xi, k_{1} x_{2}, t\right)=\frac{1}{\pi} \int_{-\infty}^{+\infty} \frac{k_{1} x_{2}}{(r-\xi)^{2}+k_{1}^{2} x_{2}^{2}} \psi_{1}^{*}(r, 0, t) d r$.

(21)

Let us once again present the results in sequence for all three considered regimes.

\subsection{Sub-Rayleigh regime}

On substituting Eq. (18) into Eq. (21), after integration we get $\psi_{1}^{*}\left(\xi, k_{1} x_{2}, t\right)=\frac{1}{\pi} \int_{-\infty}^{+\infty} \frac{k_{1} x_{2}}{(r-\xi)^{2}+k_{1}^{2} x_{2}^{2}} \psi_{1}^{*}(r, 0, t) d r$.

where

$h\left(\xi, k_{1} x_{2}, \xi_{i}\right)=\frac{k_{1} x_{2}}{2} \ln \frac{\left(\xi-\xi_{i}\right)^{2}+k_{1}^{2} x_{2}^{2}}{\xi^{2}+k_{1}^{2} x_{2}^{2}}+\xi \tan ^{-1} \frac{\xi}{k_{1} x_{2}}-(\xi-$

$\left.\xi_{i}\right) \tan ^{-1} \frac{\xi-\xi_{i}}{k_{1} x_{2}}(i=1,2)$,

and $\xi_{1}=-v_{-} t, \xi_{2}=-v_{+} t$.

Displacements (5) in terms of $\psi_{1}^{*}$ take the following form

$u_{1}=-k_{1} \frac{\partial \psi_{1}^{*}\left(\xi, k_{1} x_{2}, t\right)}{\partial \xi}-\vartheta k_{2} \frac{\partial \psi_{1}^{*}\left(\xi, k_{2} x_{2}, t\right)}{\partial \xi}$,

$u_{2}=-\frac{1}{k_{1}} \frac{\partial \psi_{1}^{*}\left(\xi, k_{1} x_{2}, t\right)}{\partial x_{2}}-\frac{\vartheta}{k_{2}} \frac{\partial \psi_{1}^{*}\left(\xi, k_{2} x_{2}, t\right)}{\partial x_{2}}$.

Then, on substituting Eq. (22) into Eq. (23), the transient displacements are written explicitly as:

$u_{1}=\frac{c_{R}^{2} P_{1}}{\pi v_{-} v_{+}}\left[k_{1} \tan ^{-1} \frac{\xi}{k_{1} x_{2}}+\vartheta k_{2} \tan ^{-1} \frac{\xi}{k_{2} x_{2}}\right]+$

$\frac{c_{R} P_{1}}{2 \pi v_{+}}\left[k_{1} \tan ^{-1} \frac{\xi-\xi_{2}}{k_{1} x_{2}}+\vartheta k_{2} \tan ^{-1} \frac{\xi-\xi_{2}}{k_{2} x_{2}}\right]-$

$\frac{c_{R} P_{1}}{2 \pi v_{-}}\left[k_{1} \tan ^{-1} \frac{\xi-\xi_{1}}{k_{1} x_{2}}+\vartheta k_{2} \tan ^{-1} \frac{\xi-\xi_{1}}{k_{2} x_{2}}\right]$ 
$u_{2}=-\frac{c_{R} P_{1}}{4 \pi v_{+}}\left[\ln \frac{\left(\xi-\xi_{2}\right)^{2}+k_{1}^{2} x_{2}^{2}}{\xi^{2}+k_{1}^{2} x_{2}^{2}}+\vartheta \ln \frac{\left(\xi-\xi_{2}\right)^{2}+k_{2}^{2} x_{2}^{2}}{\xi^{2}+k_{2}^{2} x_{2}^{2}}\right]+$
$\frac{c_{R} P_{1}}{4 \pi v_{-}}\left[\ln \frac{\left(\xi-\xi_{1}\right)^{2}+k_{1}^{2} x_{2}^{2}}{\xi^{2}+k_{1}^{2} x_{2}^{2}}+\vartheta \ln \frac{\left(\xi-\xi_{1}\right)^{2}+k_{2}^{2} x_{2}^{2}}{\xi^{2}+k_{2}^{2} x_{2}^{2}}\right]$.

We represent Eq. (24) as:

$u_{1}=u_{1}^{s t}\left(\xi, x_{2}\right)+u_{1}^{r 0}$,

where $u_{1}^{\text {st }}$ corresponds to the steady-state displacement $\left(16_{1}\right)$, and:

$u_{1}^{r 0}=\frac{c_{R} P_{1} v\left(k_{1}+\vartheta k_{2}\right)}{2 v_{-} v_{+}}$

is associated with the rigid body motion of the half-space, determined from the analysis of the limiting behaviour of displacements as $t \rightarrow \infty$.

Similarly, equation (25) can be reduced to

$u_{2}=u_{2}^{s t}\left(\xi, x_{2}\right)+u_{2}^{r 0}+u_{2}^{r 1} \ln t$,

where $u_{2}^{s t}$ corresponds to formula (162), and

$u_{2}^{r 0}=-\frac{c_{R} P_{1}(1+\vartheta)}{2 \pi}\left[\frac{\ln v_{+}}{v_{+}}-\frac{\ln \left|v_{-}\right|}{v_{-}}\right]$

$u_{2}^{r 1}=\frac{c_{R}^{2} P_{1}(1+\vartheta)}{\pi v_{-} v_{+}}$.

Thus, explicit expressions for rigid body motion have been obtained. It also follows from Eq. (29) that the vertical rigid body motion had a logarithmic growth in time, which is consistent with the previous results for isotropic elasticity in (Kaplunov et al., 2010a).

\subsection{Super-Rayleigh regime}

On substituting Eq. (19) into Eq. (21) and performing straightforward manipulations, we deduce the following equation:

$$
\begin{aligned}
& \psi_{1}^{*}\left(\xi, k_{1} x_{2}, t\right)=-\frac{c_{R} P_{1}}{2 \pi}\left[\frac { 2 c _ { R } } { v _ { - } v _ { + } } \left(\frac{k_{1} x_{2}}{2} \ln \frac{\left(\xi-\xi_{1}\right)^{2}+k_{1}^{2} x_{2}^{2}}{\xi^{2}+k_{1}^{2} x_{2}^{2}}+\right.\right. \\
& \left.\xi \tan ^{-1} \frac{\xi}{k_{1} x_{2}}-\xi \tan ^{-1} \frac{\xi-\xi_{1}}{k_{1} x_{2}}\right)-\frac{1}{c_{R}+v}\left(\frac{k_{1} x_{2}}{2} \ln \frac{\left(\xi-\xi_{2}\right)^{2}+k_{1}^{2} x_{2}^{2}}{\left(\xi-\xi_{1}\right)^{2}+k_{1}^{2} x_{2}^{2}}+\right. \\
& \left.\left.\left(\xi-\xi_{2}\right) \tan ^{-1} \frac{\xi-\xi_{1}}{k_{1} x_{2}}-\left(\xi-\xi_{2}\right) \tan ^{-1} \frac{\xi-\xi_{2}}{k_{1} x_{2}}\right)\right] .
\end{aligned}
$$

Then, from Eq. (23), the displacements $u_{1}$ and $u_{2}$ are obtained, coinciding with those for the sub-Rayleigh regime, namely Eqs (24) and (25). Study of the limiting behaviour of both displacements also gives the same structure as in Eqs (26)-(29), except for $u_{1}^{r 0}$ that now is determined by-

$u_{1}^{r 0}=-\frac{c_{R}^{2} P_{1}\left(k_{1}+\vartheta k_{2}\right)}{2 v_{-} v_{+}}$.

\subsection{Resonant regime}

Here, we study the case when the speed of the moving load coincides with the surface wave speed. Taking into account Eq. (20), the potential $\psi_{1}^{*}$ is found as:

$$
\begin{aligned}
& \psi_{1}^{*}\left(\xi, k_{1} x_{2}, t\right)=\frac{P_{1}}{4 \pi}\left[\frac{k_{1} x_{2}}{2} \ln \frac{\left(\xi+2 c_{R} t\right)^{2}+k_{1}^{2} x_{2}^{2}}{\xi^{2}+k_{1}^{2} x_{2}^{2}}+(\xi+\right. \\
& \left.\left.2 c_{R} t\right)\left(\tan ^{-1} \frac{\xi}{k_{1} x_{2}}-\tan ^{-1} \frac{\xi+2 c_{R} t}{k_{1} x_{2}}\right)\right] .
\end{aligned}
$$

$$
\begin{aligned}
& u_{1}=-\frac{c_{R} P_{1} x_{2} t}{2 \pi}\left[\frac{k_{1}^{2}}{\xi^{2}+k_{1}^{2} x_{2}^{2}}+\frac{\vartheta k_{2}^{2}}{\xi^{2}+k_{2}^{2} x_{2}^{2}}\right]-\frac{P_{1} k_{1}}{4 \pi}\left[\tan ^{-1} \frac{\xi}{k_{1} x_{2}}-\right. \\
& \left.\tan ^{-1} \frac{\xi+2 c_{R} t}{k_{1} x_{2}}\right]-\frac{P_{1} \vartheta k_{2}}{4 \pi}\left[\tan ^{-1} \frac{\xi}{k_{2} x_{2}}-\tan ^{-1} \frac{\xi+2 c_{R} t}{k_{2} x_{2}}\right] \\
& u_{2}=\frac{c_{R} P_{1} \xi t}{2 \pi}\left[\frac{1}{\xi^{2}+k_{1}^{2} x_{2}^{2}}+\frac{\vartheta}{\xi^{2}+k_{2}^{2} x_{2}^{2}}\right]-\frac{P_{1}}{8 \pi}\left[\ln \frac{\left(\xi+2 c_{R} t\right)^{2}+k_{1}^{2} x_{2}^{2}}{\xi^{2}+k_{1}^{2} x_{2}^{2}}+\right. \\
& \left.\vartheta \ln \frac{\left(\xi+2 c_{R} t\right)^{2}+k_{2}^{2} x_{2}^{2}}{\xi^{2}+k_{2}^{2} x_{2}^{2}}\right] .
\end{aligned}
$$

The limiting behavior of displacements as $t \rightarrow \infty$ for resonant case gives

$u_{1}=-\frac{c_{R} P_{1} x_{2} t}{2 \pi}\left[\frac{k_{1}^{2}}{\xi^{2}+k_{1}^{2} x_{2}^{2}}+\frac{\vartheta k_{2}^{2}}{\xi^{2}+k_{2}^{2} x_{2}^{2}}\right]$,

and:

$u_{2}=\frac{c_{R} P_{1} \xi t}{2 \pi}\left[\frac{1}{\xi^{2}+k_{1}^{2} x_{2}^{2}}+\frac{\vartheta}{\xi^{2}+k_{2}^{2} x_{2}^{2}}\right]-\frac{P_{1}(1+\vartheta)}{4 \pi} \ln \left(2 c_{R} t\right)$.

\section{NUMERICAL ILLUSTRATIONS}

First, let us illustrate the obtained steady-state solution available in Eq. (16). Three forms of the strain-energy function are considered in the following, namely the neo-Hookean, Gent, and Gent-Gent models, which are typically used for modelling rubberlike materials.

The well-known neo-Hookean strain-energy function is written as:

$W=\frac{\mu}{2}\left(I_{1}-3\right)$,

where $\mu$ is the ground-state shear modulus, and $I_{1}$ is given by:

$I_{1}=\lambda_{1}^{2}+\lambda_{2}^{2}+\lambda_{3}^{2}$,

where $\lambda_{i}(i=\overline{1,3})$ are the principal stretches of the underlying deformation, related by an incompressibility condition $\lambda_{1} \lambda_{2} \lambda_{3}=$ 1 [see Dowaikh and Ogden (1990) for more details].

Consider also extensions of the neo-Hookean model, including the Gent strain-energy function (Gent, 1996)

$W=-\frac{\mu}{2} J_{m} \ln \left(1-\frac{I_{1}-3}{J_{m}}\right)$,

where $J_{m}$ is a material constant characterising material extensibility and the shear modulus $\mu=\mu_{0}+\frac{2 C_{2}}{3}$ with $C_{2}$ denoting the material constant, along with its more advanced version usually referred to as the Gent-Gent material model (Pucci and Saccomandi, 2002) defined by

$W=-\frac{\mu_{0}}{2} J_{m} \ln \left(1-\frac{I_{1}-3}{J_{m}}\right)+C_{2} \ln \left(\frac{I_{2}}{3}\right)$,

where $I_{1}$ is given by (38), and $I_{2}=\lambda_{1}^{-2}+\lambda_{2}^{-2}+\lambda_{3}^{-2}$, see also a recent contribution by (Zhou et al., 2018).

Figs. 2, 3 demonstrate the computation results for different forms of the strain-energy function at the depth $x_{2}=0.5$. The system parameters are chosen as follows: $\mu_{0}=0.2853 \mathrm{MPa}$, $C_{2}=0.1898 \mathrm{MPa}, J_{m}=88.43$ (according to (Zhou et al., 2018)), $\rho=1522 \mathrm{~kg} / \mathrm{m}^{3}, v=0.8 c_{R}, P_{0}=1, \lambda_{1}=1.25$, $\lambda_{2}=\lambda_{1}^{-1}, \lambda_{3}=1$. It is also assumed that a parameter $Z=0$, which is used for calculating the normal Cauchy stress $\sigma_{2}$ (Dowaikh and Ogden, 1990),

$\sigma_{2}=\gamma-\sqrt{\gamma \alpha}+Z \sqrt{2 \sqrt{\gamma \alpha}(\beta+\sqrt{\gamma \alpha})}, \quad-1 \leq Z \leq 1$, ised.

Thus, the surface wave has a non-zero velocity and is local- 


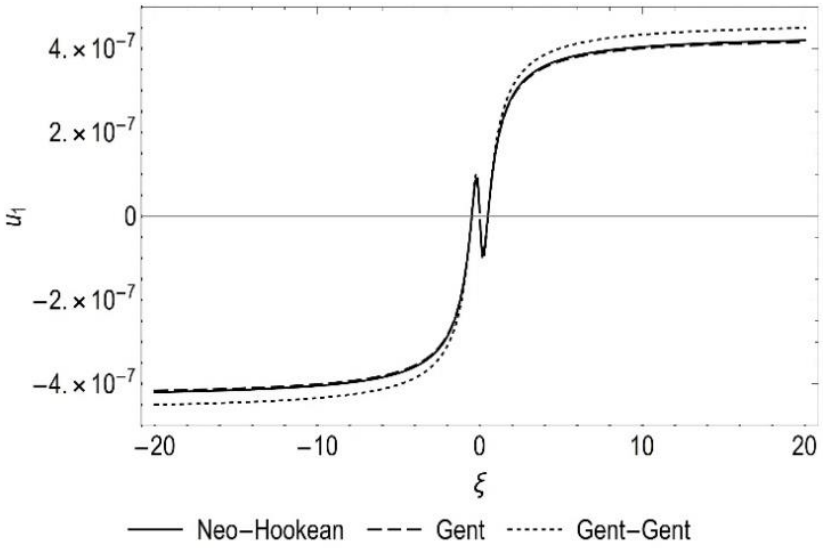

Fig. 2. Horizontal displacement $u_{1}$ for the neo-Hookean, Gent and Gent-Gent material models

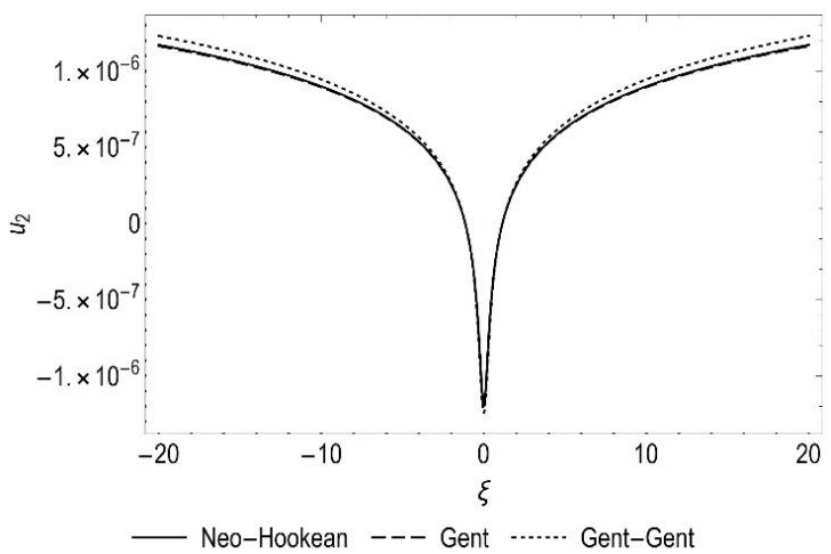

Fig. 3. Vertical displacement $u_{2}$ for the neo-Hookean, Gent and Gent-Gent material models

As can be seen from Figs 2 and 3, the neo-Hookean and Gent material models give almost identical results for both displacements $u_{1}$ and $u_{2}$, whereas using the Gent-Gent model results in higher values of the displacement amplitudes with increasing $\xi$. In what follows, we use the Gent-Gent model, since it seemingly provides a better agreement with experimental data, as demonstrated in a study by Zhou et al. (2018).

Variation of the amplitude of the surface displacements $u_{1}$ and $u_{2}$ on the moving coordinate is illustrated in Figs 4 and 5 for several values of the transverse variable $x_{2}$.

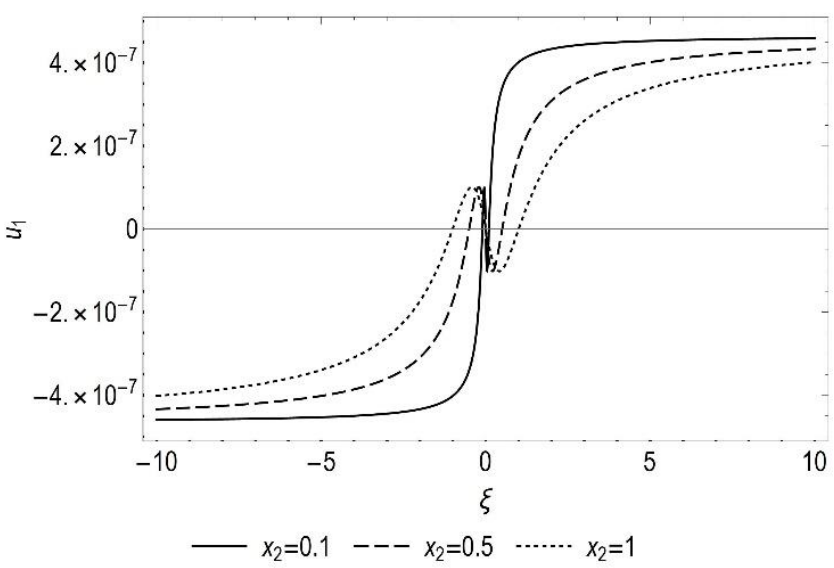

Fig. 4. Dependence of displacement $u_{1}$ on the moving coordinate $\xi$ for different values of $x_{2}$

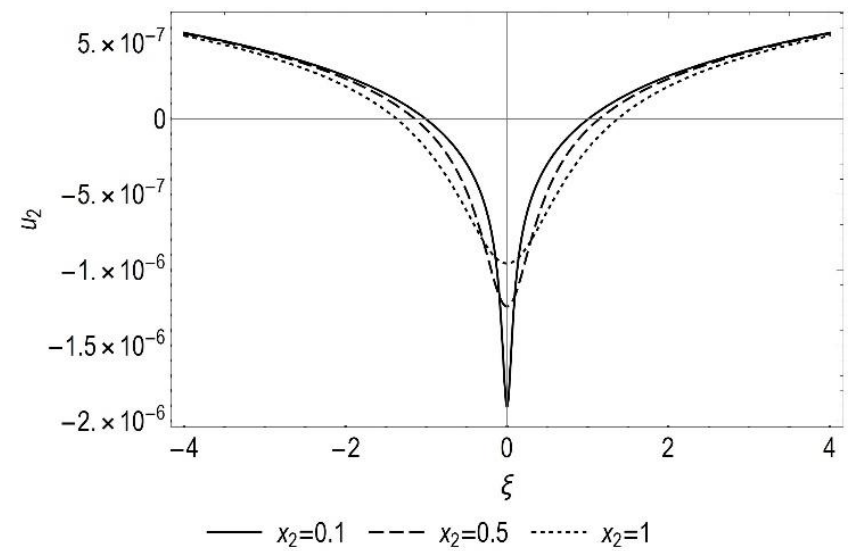

Fig. 5. Dependence of displacement $u_{2}$ on the moving coordinate $\xi$ for different values of $x_{2}$

Predictably, the obtained graphs (Figs 4 and 5) indicate smoothening of displacement profiles under the moving load with increase in depth.

To present the numerical results for the transient moving load problem, the Gent-Gent model [Eq. (40)] is used for the same material parameters as before. The speed of the moving load for various regimes is taken as $v=0.8 c_{R}, v=1.2 c_{R}$ and $v=c_{R}$ for the sub-Rayleigh, super-Rayleigh and resonant regimes, respectively (Figs 6-11).

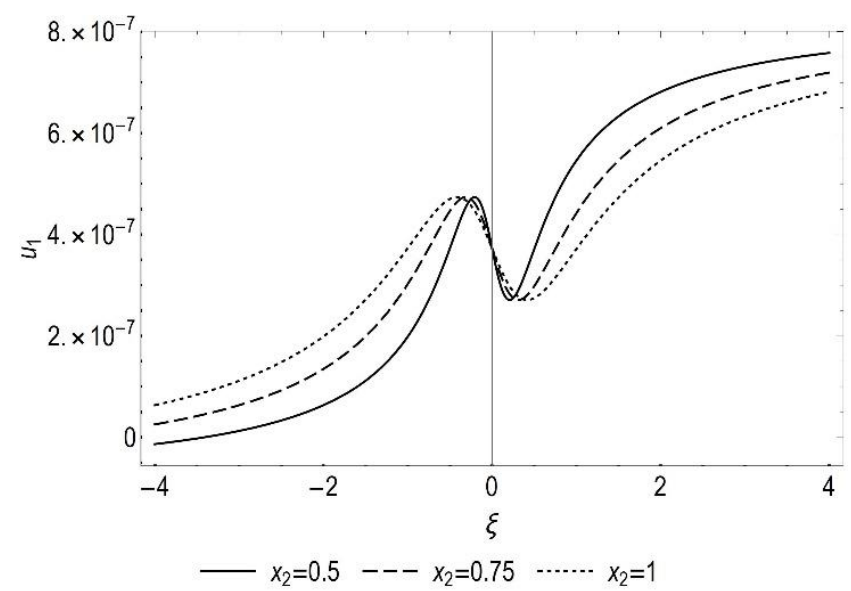

Fig. 6. The sub-Rayleigh transient displacement $u_{1}$ for different values of $x_{2}$

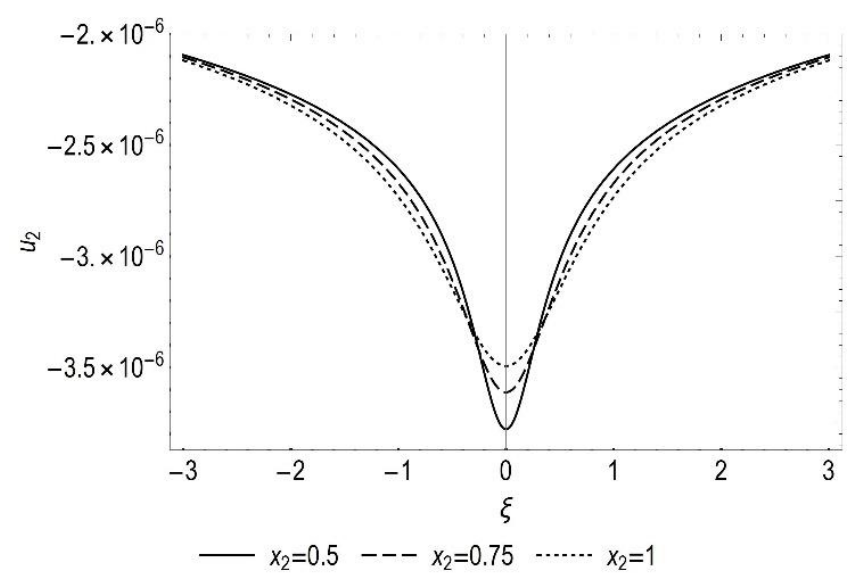

Fig. 7. The sub-Rayleigh transient displacement $u_{2}$ for different values of $x_{2}$ 
Figs 6 and 7 demonstrate the sub-Rayleigh transient displacements $\mathrm{u}_{1}$ and $\mathrm{u}_{2}$; Figs. 8 and 9 represent the superRayleigh transient displacements, see Eqs (24) and (25); and Figs. 10 and 11 correspond to the resonant transient displacements Eqs (33) and (34), depending on the values of the vertical coordinate $\mathrm{x}_{2}$.

It is emphasised that the obtained shapes of displacements are typical for a broad range of deformations, with qualitatively similar behaviour occurring for the non-deformed linear isotropic case as well, when $\lambda_{1}=\lambda_{2}=\lambda_{3}=1$.

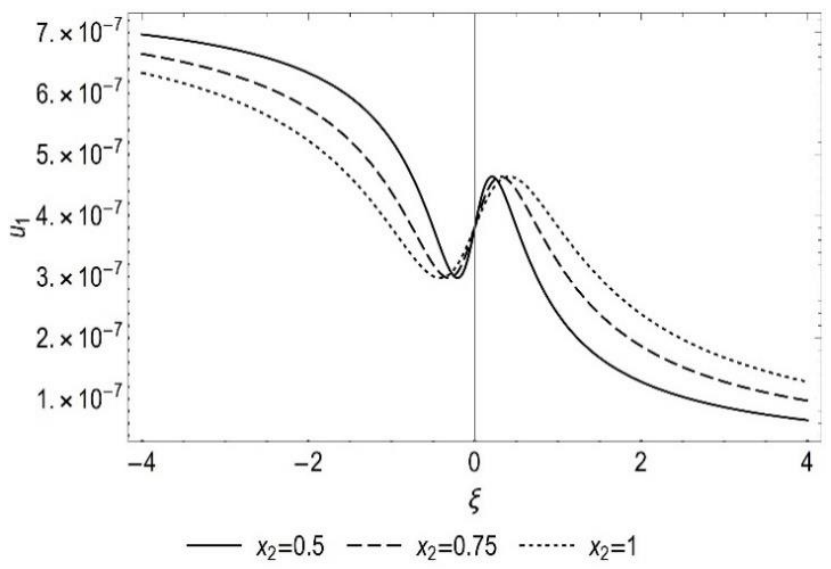

Fig. 8. The super-Rayleigh transient displacement $u_{1}$ for different values of $x_{2}$

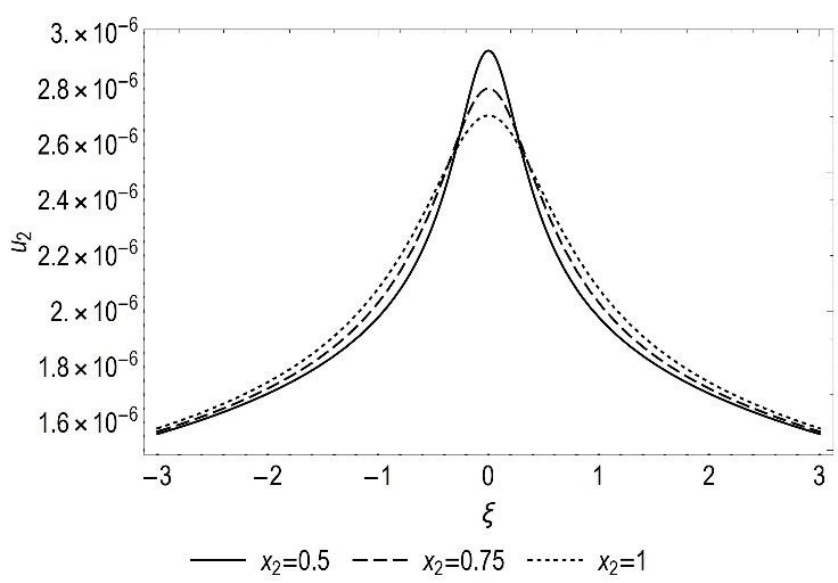

Fig. 9. The super-Rayleigh transient displacement $u_{2}$ for different values of $x_{2}$

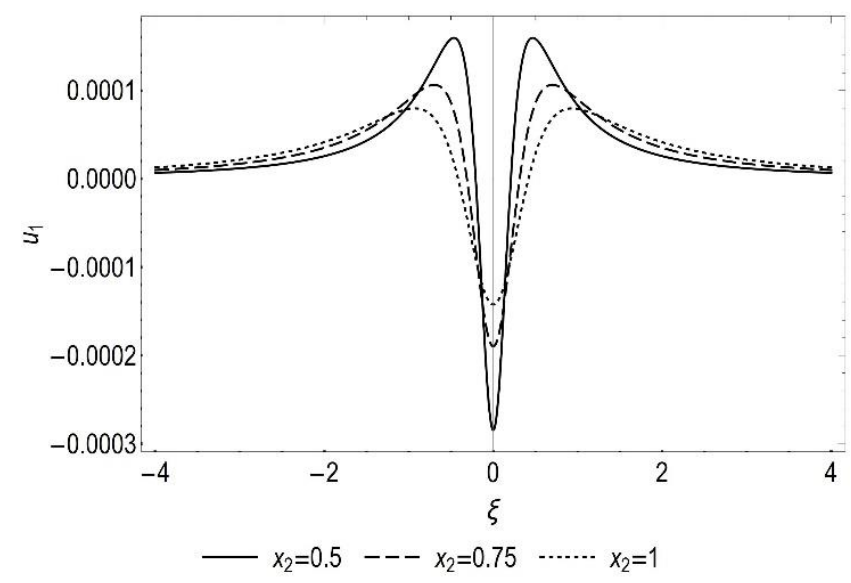

Fig. 10. The resonant transient displacement $u_{1}$ for different values of $x_{2}$

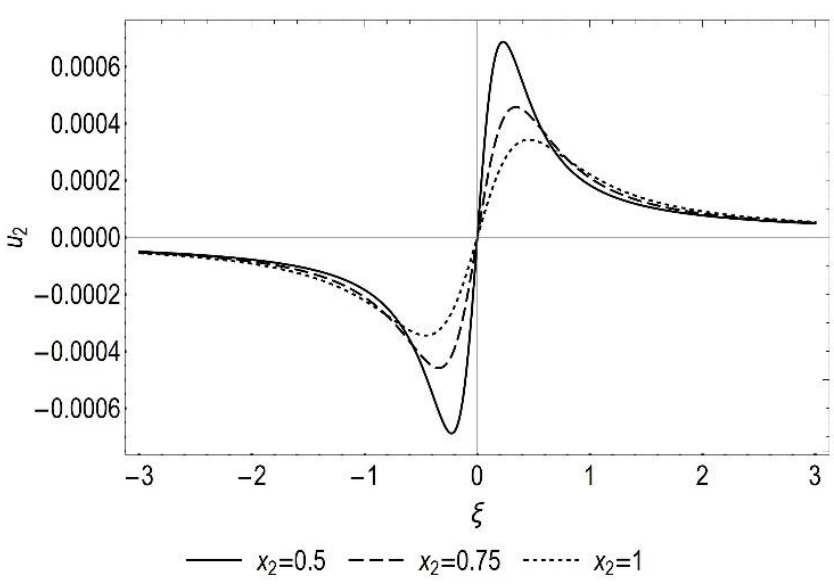

Fig. 11. The resonant transient displacement $u_{2}$ for different values of $x_{2}$

It should be noted that the accuracy of the approximate results within the model has been discussed in a study by (Kaplunov and Prikazchikov, 2017; Sect. 4.3.1), where it was shown that for a wide class of loads, the asymptotic formulation captures the contribution of the Rayleigh poles. Moreover, in case of transient problem, as shown in a study by Kaplunov et al. (2010a), the near-resonant solution is valid for large times when the effect of the body waves becomes negligible. Moreover, comparison of exact and approximate solutions revealed a wide range of speeds, in which the approximation performs at a reasonable accuracy [Fig. 8 in Kaplunov et al. (2010a)].

\section{CONCLUSION}

The near-resonant regimes of the steady-state moving load problem on a pre-stressed, incompressible elastic half-space have been studied. Implementation of the hyperbolic-elliptic model for surface wave allowed explicit solutions for the displacement components. Illustrations in Figs. 2 and 3 revealed some possible similarities between the neo-Hookean, Gent, and Gent-Gent material models. The consideration has then been extended to transient problem, allowing an elegant approximate solution in terms of elementary functions, which makes it convenient for further analysis, including the limiting behaviour for large time, providing explicit results for the components of rigid body motion.

Various extensions of the approach include derivation of 3D asymptotic models in pre-stressed media. Although a straightforward explicit approach could be cumbersome algebraically, there is a chance of more elegant representation though Stroh formalism, in line with results reported recently in a study by Fu et al. (2020). It is worth noting that adding vertical inhomogeneity will lead to smoothing of surface discontinuities; for more details refer to a study by Erbaş et al. (2017). Finally, we note that it is also possible to extend the methodology to composite models for elastic layers (Erbaş et al., 2018; Erbaş et al., 2019), as well as to consider the dynamics of a pre-stressed half-space with cavities (Alekseeva and Ukrainets, 2009) and crack propagation (Mishuris et al., 2012; Gourgiotis and Piccolroaz, 2014).

\section{REFERENCES}

1. Alekseeva L.A., Ukrainets V.N. (2009), Dynamics of an elastic halfspace with a reinforced cylindrical cavity under moving loads, Int. Appl. Mech., 45(9), 981-990. 
2. Bratov V. (2011), Incubation time fracture criterion for FEM simulations, Acta Mech. Sin., 27(4), 541.

3. Cao Y., Xia H., Li Z. (2012), A semi-analytical/FEM model for predicting ground vibrations induced by high-speed train through continuous girder bridge, J. Mech. Sci. Technol., 26, 2485-2496.

4. Cole J., Huth J. (1958), Stresses produced in a half plane by moving loads, J. Appl. Mech., 25, 433-436.

5. de Hoop A.T. (2002), The moving-load problem in soil dynamics the vertical displacement approximation, Wave Motion, 36(4), 335346.

6. Dimitrovová Z. (2017), Analysis of the critical velocity of a load moving on a beam supported by a finite depth foundation, Int. J. Solids Struct., 122, 128-147

7. Dowaikh M.A., Ogden R.W. (1990), On surface waves and deformations in a pre-stressed incompressible elastic solid, IMA J. Appl. Math., 44, 261-284.

8. Ege N., Erbaş B., Kaplunov J., Wootton P. (2018), Approximate analysis of surface wave-structure interaction, J. Mech. Mater. Struct., 13(3), 297-309.

9. Ege N., Şahin O., Erbaş B. (2017), Response of a 3D elastic halfspace to a distributed moving load, Hacet J. Math. Stat., 46(5), 817 828.

10. Erbaş B., Kaplunov J., Nolde E., Palsü M. (2018), Composite wave models for elastic plates, P. Roy. Soc. A-Math. Phy., 474(2214), 116.

11. Erbaş B., Kaplunov J., Palsü M. (2019), A composite hyperbolic equation for plate extension, Mech. Res. Commun., 99, 64-67.

12. Erbaş B., Kaplunov J., Prikazchikov D.A., Şahin O. (2017), The near-resonant regimes of a moving load in a three-dimensional problem for a coated elastic half-space, Math. Mech. Solids, 22(1), 89100.

13. Fryba L. (1999), Vibration of solids and structures under moving loads, 3rd ed, Thomas Telford, London.

14. Fu Y., Kaplunov J., Prikazchikov D. (2020), Reduced model for the surface dynamics of a generally anisotropic elastic half-space, $P$. Roy. Soc. A-Math. Phy., 476(2234), 1-19.

15. Gakenheimer D.C., Miklowitz J. (1969), Transient excitation of an elastic half space by a point load traveling on the surface, J. Appl. Mech., 36(3), 505-515.

16. Gent A.N. (1996), A new constitutive relation for rubber, Rubber Chem. Technol., 69(1), 59-61.

17. Goldstein R.V. (1965), Rayleigh waves and resonance phenomena in elastic bodies, J. Appl. Math. Mech. (PMM), 29(3), 516-525.

18. Gourgiotis P.A., Piccolroaz A. (2014), Steady-state propagation of a mode II crack in couple stress elasticity, Int. J. Fract., 188(2), 119145 .

19. Kaplunov J., Nolde E., Prikazchikov D.A. (2010a), A revisit to the moving load problem using an asymptotic model for the Rayleigh wave, Wave Motion, 47, 440-451.

20. Kaplunov J., Prikazchikov D., Sultanova L. (2019), Rayleigh-type waves on a coated elastic half-space with a clamped surface, Phil. Trans. Roy. Soc. A, 377(2156), 1-15.

21. Kaplunov J., Prikazchikov D.A. (2017), Asymptotic theory for Rayleigh and Rayleigh-type waves, Adv. Appl. Mech., 50, 1-106.

22. Kaplunov J., Prikazchikov D.A., Erbaş B., Şahin O. (2013), On a 3D moving load problem for an elastic half space, Wave Motion, 50(8), 1229-1238

23. Kaplunov J., Voloshin V., Rawlins A.D. (2010b), Uniform asymptotic behaviour of integrals of Bessel functions with a large parameter in the argument, Quart. J. Mech. Appl. Math., 63(1), 57-72.
24. Khajiyeva L.A., Prikazchikov D.A., Prikazchikova L.A. (2018), Hyperbolic-elliptic model for surface wave in a pre-stressed incompressible elastic half-space, Mech. Res. Commun., 92, 49-53.

25. Krylov V.V. (1996), Vibrational impact of high-speed trains. I. Effect of track dynamics, J. Acoust. Soc. Am., 100(5), 3121-3134.

26. Kumar R., Vohra R. (2020), Steady state response due to moving load in thermoelastic material with double porosity, Mater. Phys. Mech., 44(2), 172-185.

27. Lefeuve-Mesgouez G., Le Houédec D., Peplow A.T. (2000), Ground vibration in the vicinity of a high-speed moving harmonic strip load, J. Sound Vib., 231(5), 1289-1309.

28. Lu T., Metrikine A.V., Steenbergen M.J.M.M. (2020), The equivalent dynamic stiffness of a visco-elastic half-space in interaction with a periodically supported beam under a moving load, Europ. J. Mech.A/Solids, 84, 104065

29. Mishuris G., Piccolroaz A., Radi E. (2012), Steady-state propagation of a Mode III crack in couple stress elastic materials, Int. J. Eng. Sci., 61, 112-128.

30. Ogden R.W. (1984), Non-linear elastic deformations, Dover, New York.

31. Payton R.G. (1967), Transient motion of an elastic half-space due to a moving surface line load, Int. J. Eng. Sci., 5(1), 49-79.

32. Pucci E., Saccomandi G. (2002), A note on the Gent model for rubber-like materials, Rubber Chem. Technol., 75(5), 839-852.

33. Smirnov V., Petrov Yu.V., Bratov V. (2012), Incubation time approach in rock fracture dynamics, Sci. China Phys., Mech. Astr., 55(1), 78-85.

34. Sun Z., Kasbergen C., Skarpas A., Anupam K., van Dalen K.N., Erkens S.M. (2019), Dynamic analysis of layered systems under a moving harmonic rectangular load based on the spectral element method, Int. J. Solids Struct., 180, 45-61.

35. van Dalen K.N., Tsouvalas A., Metrikine A.V., Hoving J.S. (2015), Transition radiation excited by a surface load that moves over the interface of two elastic layers, Int. J. Solids Struct., 73, 99-112.

36. Wang F., Han X., Ding T. (2021), An anisotropic layered poroelastic half-space subjected to a moving point load, Soil Dyn. Earth. Eng., 140,106427

37. Wang Y., Zhou A., Fu T., Zhang W. (2020), Transient response of a sandwich beam with functionally graded porous core traversed by a non-uniformly distributed moving mass, Int. J. Mech. Mater. Design, 16(3), 519-540.

38. Wootton P.T., Kaplunov J., Colquitt D.J. (2019), An asymptotic hyperbolic-elliptic model for flexural-seismic metasurfaces, P. Roy. Soc. A-Math. Phy., 475(2227), 1-18.

39. Wootton P.T., Kaplunov J., Prikazchikov D. (2020), A secondorder asymptotic model for Rayleigh waves on a linearly elastic half plane, IMA J. Appl. Math., 85, 113-131.

40. Zhou L., Wang S., Li L., Fu Y. (2018), An evaluation of the Gent and Gent-Gent material models using inflation of a plane membrane, Int J. Mech. Sci., 146-147, 39-48.

Acknowledgements: Askar $\mathrm{K}$ and Askat $\mathrm{K}$ acknowledge support from the Ministry of Science and Education of the Republic of Kazakhstan (Grant No. AP05132743, 2018-2020). DP was supported by the Russian Science Foundation, Grant No. 20-11-20133 in the part on "Explicit steadystate solution for the near-resonant regime". 